\title{
MicroRNA-138 Aggravates Inflammatory Responses of Macrophages by Targeting SIRT1 and Regulating the NF-KB and AKT Pathways
}

\author{
Xiao-Zhi Bai $\quad$ Ju-Lei Zhanga ${ }^{a}$ Yang Liu ${ }^{a} \quad$ Wei Zhang ${ }^{a} \quad$ Xiao-Qiang Lia \\ Ke-Jia Wang ${ }^{a}$ Meng-Yuan Cao ${ }^{b}$ Jia-Ning Zhang ${ }^{c}$ Fu Han ${ }^{a} \quad$ Ji-Hong Shi ${ }^{a}$ \\ Da-Hai Hua
}

aDepartment of Burns and Cutaneous Surgery, Xijing Hospital, the Fourth Military Medical University, Xi'an, ${ }^{b}$ Department of Emergency, Xijing Hospital, the Fourth Military Medical University Xi'an, '1st Cadet Brigade, the Fourth Military Medical University Xi'an, China

\section{Key Words}

Inflammation $\cdot$ MicroRNA-138 $\cdot \mathrm{SIRT} 1 \cdot$ Macrophage $・ N F-K B \cdot A K T$

\begin{abstract}
Background/Aims: With increased understanding of sepsis, mortality is decreasing. However, there is still a lack of effective therapeutic strategy. The inflammatory response of macrophages is critical during sepsis. Methods: Macrophages were stimulated with LPS. Western blotting and qRT-PCR were used to detect inflammatory responses. Then, the inhibitor of microRNA-138 was transfected and Western blotting, GRT-PCR, H\&E staining and ELISA were used to verify the role of microRNA-138 in inflammation. Then target gene prediction databases were used to predict the potential target of microRNA-138. Both animal and cell models under LPS challenges were established to verify the regulation of SIRT1 and microRNA-138 during inflammation. Results: The present study showed that microRNA-138 was increased in macrophages stimulated with LPS. Additionally, the NF-KB and AKT pathways were both activated. The pre-treatment of microRNA-138 inhibitor decreased inflammatory factors, downregulated the NF-KB pathway, activated the AKT pathway and protected against organ damage in mice challenged with LPS. SIRT1 was demonstrated as a potential target of microRNA-138In macrophages stimulated with LPS, the inhibition effect of microRNA-138 inhibitor on inflammation was lost by SIRT1 siRNA pre-treatment. In the animal model, the protective effect of microRNA-138 antagomir disappeared in SIRT1 knockout mice. Conclusion: We demonstrated that miR-138 participated in the inflammatory process by inhibiting SIRT1 and activating the NF-KB pathway.

X.-Z. Bai, J-L. Zhang and Y. Liu contributed equally to this work.

\begin{tabular}{ll}
\hline Dahai Hu & Department of Burns and Cutaneous Surgery, Xijing Hospital, the Fourth Military Medical University \\
& 127 Changle West Road, Xi'an, Shaanxi 710032 (China) \\
& Tel. +86-29-8477 5298, Fax +86-29-83251734, E-Mail xjburnlab@163.com
\end{tabular}




\section{Cellular Physiology Cell Physiol Biochem 2018;49:489-500

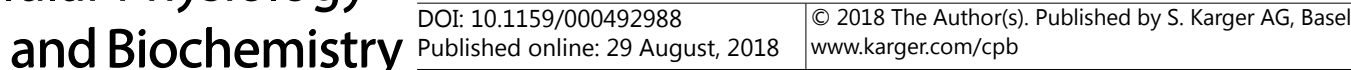

Bai et al.: MicroRNA-138 Promotes Inflammation Through SIRT1

\section{Introduction}

Sepsis, which is caused by a disorganized response to infection, is currently characterized by severe inflammatory reaction and immune suppression. Despite the successful supportive therapies and decreasing mortality [1], there is still a lack of targets for earlier treatment $[2,3]$. Owing to the severe inflammation, sepsis often causes tissue damage and organ dysfunction, which determine the presenting symptoms of sepsis [2]. The affected tissue often shows increased infiltration of inflammatory cells, edema, hemorrhage and damage of the tissue structure [4]. Macrophages are important components of the innate immune system that modulate immune responses through the recognition and presentation of antigens and the release of inflammatory factors [5]. The activation of macrophages is related to many diseases and plays a key role in the process of sepsis by participating in inflammation and immune suppression [6]. Depending on different circumstances, macrophages may differentiate into two different phenotypes. M1 macrophages (or classically activated macrophage) are pro-inflammatory macrophages that release IL-1 $\beta$, TNF- $\alpha$, IL- 6 and other inflammatory factors under the stimulation of IFN- $\gamma$ or lipopolysaccharide (LPS) through the activation of the STAT1 or NF- $\kappa$ B pathway $[7,8]$. These inflammatory factors are also important cytokines increased in sepsis during severe inflammatory periods. M2 macrophages (or alternatively activated macrophages) are anti-inflammatory macrophages that depend on the activation of STAT6 or AKT pathways [9, 10]. MicroRNAs (miRNAs) are non-coding RNAs that regulate gene expression by binding to mRNA, stopping translation or degrading the mRNAs. These functions are mainly achieved by the binding of mi-RNAs with the 3'-UTR of mRNAs [11]. MiRNAs have recently been implicated in inflammatory responses, sepsis, and, specifically, macrophage activities [9, 12-14]. MicroRNA-138 (miR-138) is reported to participate in oncogenesis and axon regeneration, but it is not clear whether miR-138 plays a role in inflammation or sepsis $[15,16]$. Silence information regulator 1 (SIRT1) is an NAD+ dependent class III histone deacetylase that modulates intracellular activities through transcription regulation in several separate cellular functions $[17,18]$. Recently, SIRT1 was demonstrated to inhibit inflammatory reactions 6 . Additionally, we previously showed that SIRT1 regulates the effect of melatonin on kidney protection against inflammation reactions in severely burned rats [19]. Previously study demonstrated that SIRT1 is a direct target of miR-138, participating in cancer and axon regeneration $[16,20,21]$, but whether miR138 participates in inflammation by targeting SIRT1 is still unclear. In the present study, we provided the first evidence that miR-138 is an important regulator participating in the inflammatory process and the pre-treatment of miR-138 inhibitor showed significantly effect protecting mice from LPS challenge. Additionally, we showed that miR-138 may regulate inflammatory responses by inhibiting SIRT1 and AKT pathways while activating the NF- $\kappa \mathrm{B}$ pathway.

\section{Materials and Methods}

\section{Animals}

Adult male C57BL/6 mice and myeloid-specific SIRT1 knockout (sirt1\%) mice weighing 20-25 g were included in the present study. All protocols were approved by the Ethics Committee of Xijing Hospital, affiliated with the Fourth Military Medical University (XJYYLL-2015206). The National Institutes of Health Guide for the Care and Use of Laboratory Animals (NIH Publications No. 8023, revised 1978) was followed. Mice aged six to eight weeks were used for further research. 


\section{Cellular Physiology Cell Physiol Biochem 2018;49:489-500 \begin{tabular}{l|l|l} 
and Biochemistry Published online: 29 August, 2018 & $\begin{array}{l}\text { C) 2018 The Author(s). Published by S. Karger AG, Basel } \\
\text { www.karger.com/cpb }\end{array}$ \\
\hline
\end{tabular}}

LPS induced inflammatory challenge in mice

C57BL/6 mice and sirt $1 \%$ mice were used in the present study. Briefly, the mice were intraperitoneally injected with LPS (Sigma, USA) with a dose of $10 \mathrm{mg} / \mathrm{kg}$. After sacrifice, the serum was collected at 24 hours later, and the liver, heart, kidney and lung tissue were collected at 72 hours later for further analysis. For the inhibition of miR-138, the miR-138 antagomir was injected by the caudal vein at a dose of $80 \mathrm{mg} / \mathrm{kg}$ for 3 consecutive days, followed by LPS $(10 \mathrm{mg} / \mathrm{kg})$ injection at 24 hours after the last antagomir administration.

\section{Histological examination and evaluation}

At 72 hours after LPS injection, the mice were anaesthetized with sodium pentobarbital (30 mg/kg, intraperitoneal injection) and were sacrificed; the heart, liver, lung and kidney were collected and fixed in $10 \%$ formalin for 24 hours. Then, the specimens were dehydrated, embedded in paraffin, cut into $4-\mu \mathrm{m}$ sections and stained with hematoxylin and eosin (H\&E) for light microscopy observation. For every slice, ten high-magnification fields were randomly selected and photograph by the Olympus FSX100 microscope (Japan). Then the organ damage was evaluated by pathological expert according to the scoring system 22. Briefly, the inflammatory cell infiltration, edema of alveoli and interstitial and hemorrhage were evaluated for lung damage (score 0 to 4). The liver damage degree was evaluated by hepatocyte, sinus hepaticus and converged tube (score 0 to 4). The heart tissues were evaluated according to interstitial lymphocytes infiltration, myocardial fibers, transverse striation (score 0 to 4). The kidney was evaluated according to the Glomerulus, Renal tubule and Renal interstitium damage (score 0 to 4 ).

\section{ELISA and biochemical marker detection}

At 24 hours after LPS injection, the mice were anaesthetized with sodium pentobarbital $(30 \mathrm{mg} / \mathrm{kg}$, intraperitoneal injection), the eyeballs were extracted for blood collection and the blood was coagulated at room temperature for 30 minutes. Then, the sample was centrifuged for 15 minutes at $3000 \mathrm{rpm}$, and the serum was collected. The cytokines, including IL-1 $\beta$, TNF- $\alpha$ and IL-6, were examined by using ELISA (Nanjing Jiancheng, China). ALT, AST, Cr and BUN (Nanjing Jiancheng, China) were detected to evaluate organ function.

\section{Cell culture}

The macrophage cell line RAW 264.7 was cultured in RMPI 1640 medium (Gibco, USA) containing 10\% fetal bovine serum (Gibco, USA), $100 \mathrm{IU} / \mathrm{ml}$ penicillin and $100 \mathrm{mg} / \mathrm{ml}$ streptomycin (Beyotime, Shanghai, China), and maintained at $37^{\circ} \mathrm{C}$ in a humidified incubator with $5 \% \mathrm{CO}$. For simulation of LPS challenge in vitro, RAW 264.7 cells were stimulated by LPS at a concentration of $1 \mu \mathrm{g} / \mathrm{ml}$. For the detection of signal pathways, the cells were collected at 30 minutes after stimulation. For mRNA detection, the cells were collected at 4 hours after stimulation.

Real-time quantitative polymerase chain reaction

Total RNA was extracted by using an RNA isolation kit (Takara, Japan) according to the manufacturer's instructions. The concentration and purity of the RNA were examined by the absorbance at 260 and 280 $\mathrm{nm}$ by using spectrophotometry (SmartSpecTM Plus, BIO-RAD, USA). The PrimeScript ${ }^{\mathrm{TM}}$ RT reagent Kit (Takara, Japan) was used to obtain cDNA. Real-time quantitative polymerase chain reaction (qRT-PCR) was performed by using the SYBR®Premix Ex Taq ${ }^{\text {TM }}$ Kit (Takara, Japan) in the CFX Connect (BIO-RAD, USA). The relative quantification of target gene was conducted by using the $2-^{\Delta \Delta \mathrm{Ct}}$ method. GAPDH and u6 were used as internal controls for mRNA and miRNA, respectively. The following primer sequences were used:

GAPDH-F: 5'-GTGTTCCTACCCCCAATGTG-3',

GAPDH-R: 5'-CATCGAAGGTGGAAGAGTGG-3',

IL-1 $\beta$-F:5'-GGGCCTCAAAGGAAAGAATC-3',

IL-1 $\beta$-R:5'-TACCAGTTGGGGAACTCTGC-3',

TNF- $\alpha$-F:5'-CGTCAGCCGATTTGCTATCT-3',

TNF- $\alpha-R: 5$ '-CTTGGGCAGATTGACCTCAG-3',

IL-6-F:5'-GGGACTGATGCTGGTGACAA-3',

IL-6-R:5'-TCCACGATTTCCCAGAGAACA-3',

u6: 5'-GTGCTCGCTTCGGCAGCACATAT-3',

miR-138: 5'- AGCTGGTGTTGTGAATCAGG-3'.

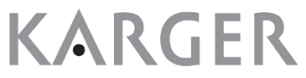




\section{Cellular Physiology Cell Physiol Biochem 2018:49:489-500

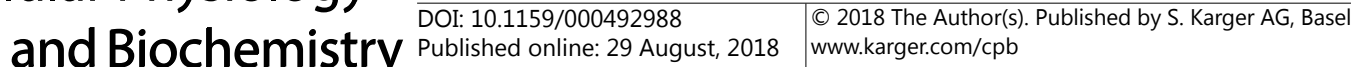

Bai et al.: MicroRNA-138 Promotes Inflammation Through SIRT1

Western blot analysis

$50 \mu \mathrm{g}$ of total protein of each sample was subjected to SDS-PAGE and transferred onto PVDF membranes. After blocking with 5\% non-fat milk, the membranes were incubated with primary antibodies, horseradish peroxidase-conjugated-conjugated secondary antibodies and visualized with an ECL Kit (Millipore, USA) by using the FluorChem FC system (Alpha Innotech, USA). The primary antibodies included anti-SIRT1, anti-p65, anti-p-p65, anti-AKT, anti-p-AKT, and anti- $\beta$-actin antibodies (all from CST, USA). The secondary antibody was HRP-conjugated goat anti-rabbit antibody (1:3000 dilution; Santa Cruz, USA)

\section{Inhibitor and siRNA transfection}

The mimic or inhibitor of miR-138, siRNA of SIRT1 and their control fragments were purchased from GeneCopoeia (China) and transfected into RAW 264.7 macrophage cell line by using Lipofectamine 2000 Transfection Reagent (Invitrogen, USA) according to the manufacturer's instructions. The cells were used for further experiments at 24 hours later. The following primer sequences were used: miR-138 inhibitor: CGGCCUGAUUCACAACACCAGCU, si-SIRT1 sense: 5'-CCGUCUCUGUGUCACAAAUTT-3', si-SIRT1 anti-sense: 5'-AUUUGUGACACAGAGACGGTT-3', control siRNA sense: 5'-UUCUCCGAACGUGUCACGUTT-3', control siRNA anti-sense: 5'-ACGUGACACGUUCGGAGAATT-3'.

\section{Luciferase reporter assay}

The target genes of miR-138 were predicted by using target prediction databases, including TargetScan, PicTar and miRanda. The plasmid contains fragment of SIRT1 3'-UTR (WT) and its mutant sequences (MUT) were synthesized by Promega (Madison, WI). Then, the plasmids were co-transfected with miR138 mimic or control miRNA into 293 cells by using Lipofectamine 2000 Transfection Reagent (Invitrogen, USA) according to the manufacturer's instructions. At 48 hours after transfection, the luciferase activity was measured by a luminometer (Promega, USA).

\section{Statistical analysis}

All data are presented as the means \pm SEM. The scoring data were analyzed using KruskalWallis analysis by SPSS 13.00. Other data were analyzed by using student's t test for two group or an analysis of variance (ANOVA) for multiple group using GraphPad Prism 5 (San Diego, USA). A p value of less than 0.05 was considered statistically significant.

\section{Results}

MiR-138 was increased in macrophages after LPS challenge

To determine the expression level of miR-138, RAW 264.7 macrophages were stimulated with LPS. Then, the mRNA level was detected by using qRTPCR. At 4 hours after stimulation, the mRNA levels of IL-1 $\beta$, TNF- $\alpha$ and IL- 6 were remarkably increased (Fig. 1A), representing the activation and proinflammatory activity of macrophages. Notably, miR-138 was also significantly increased after treatment with LPS (Fig. 1B). Additionally, LPS stimulation activated the NF- $\kappa \mathrm{B}$ pathway, as shown by the western blotting, demonstrating

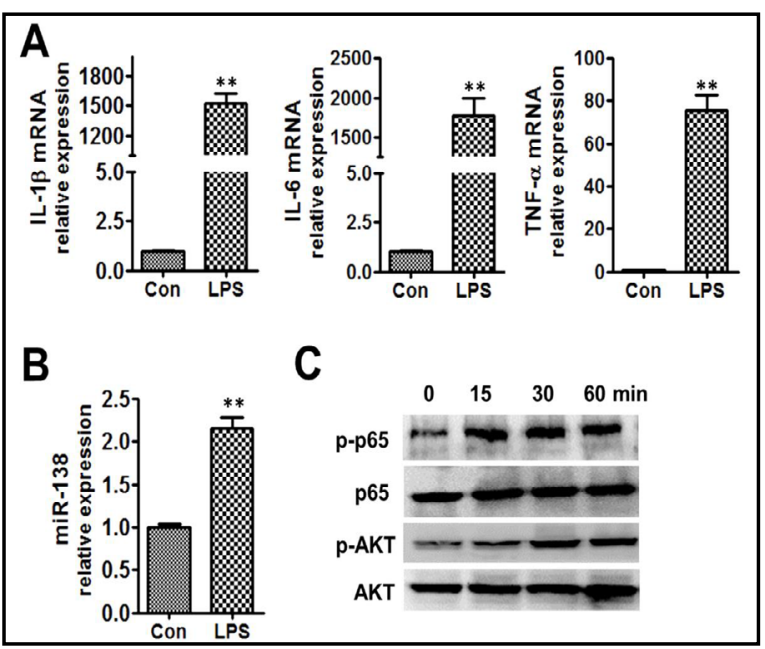

Fig. 1. MiR-138 was increased in cell model of sepsis in macrophage induced by LPS. A-C, RAW 264.7 cells were stimulated by LPS $(1 \mu \mathrm{g} / \mathrm{ml})$. At 4 hours later, relative transcript levels for IL-1 $\beta$, IL-6, TNF- $\alpha$ (A) and miR-138 (B) were detected by qRT-PCR. GAPDH and $\mathrm{u} 6$ were used as internal controls for mRNA and miRNA, respectively. $\mathrm{C}$, Representative results of the western blot showing the activation of two pathways, i.e., the AKT and NF- $\kappa B$ pathways, at different time points under LPS stimulation. For $\mathrm{A}-\mathrm{C}$, the data are the representative results of four independent experiments. Con, control group. ${ }^{* *}, \mathrm{p}<0.01$. 
Fig. 2. $I \mathrm{n} \mathrm{h}$ i b i t i o $\mathrm{n}$ of MiR-138 decreased inflammatory reaction in RAW 264.7. A, The effect of miR-138 inhibitor was verified by qRT-PCR. The results showed a strong decrease by the inhibitor. B, RAW 264.7 cells were transfected with control inhibitor (in-NC) or miR138 inhibitor (in-138) for 24 hours, followed by stimulation with LPS. At four hours later, the relative transcript level for IL-1 $\beta$, IL- 6 and TNF- $\alpha$ (B) were detected by qRT-PCR, and the activation of the two pathways, i.e., the AKT
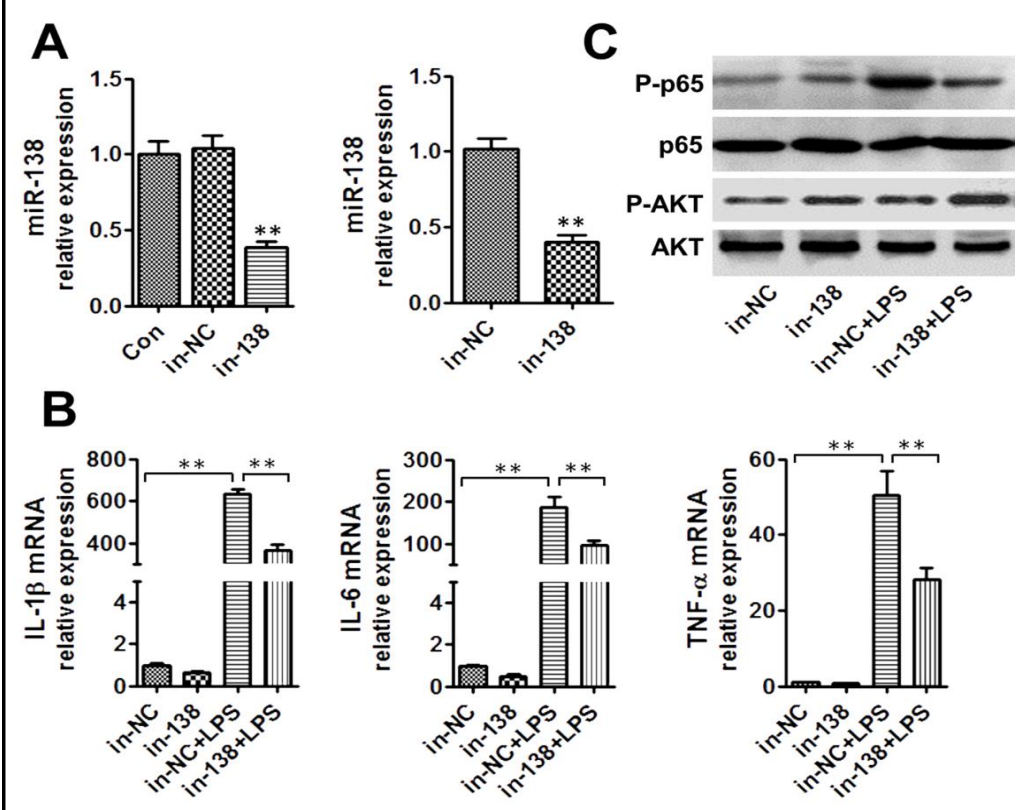
and NF- $\kappa \mathrm{B}$ pathways, was detected by western blotting at 30 minutes after LPS stimulation (C). For A-C, the data are the representative results of four independent experiments. ${ }^{* *}, \mathrm{p}<0.01$.

that p65 was phosphorylated as early as 15 minutes after stimulation. Unexpectedly, the AKT pathway was also activated, although at a later time point (Fig. 1C).

\section{Inhibition of miR-138 decreased inflammatory reaction in RAW 264.7}

To verify whether miR-138 participates in macrophage activation to a pro-inflammatory phenotype, the inhibitor of miR-138 was transfected into RAW 264.7 macrophages. As confirmed by qRT-PCR, the level of miR-138 was strongly inhibited by the inhibitor (Fig. 2A). At 24 hours after transfection, the cells were stimulated with LPS for another 4 hours. The inhibition of miR-138 significantly decreased the mRNA levels of IL-1 $\beta$, IL- 6 and TNF- $\alpha$ (Fig. $2 \mathrm{~B})$. The NF- $\kappa \mathrm{B}$ pathway was also inhibited, while the AKT pathway was strongly activated (Fig. 2C). Thus, miR-138 promoted the pro-inflammatory activation of macrophages.

\section{The miR-138 antagomir pre-treatment alleviated tissue damage and organ dysfunction} in mice

Then, we examined whether miR-138 participated in the inflammation process in vivo. Thus, mice were transfected with the miR-138 antagomir through the caudal vein, and then, the mice were subjected to LPS intraperitoneal injection. At 24 hours later, the mice were sacrificed, and the blood was collected. At 72 hours later, the tissue was collected for further analysis. The sections of the tissue were stained with H\&E for pathological analysis. H\&E staining of the heart, liver, lung and kidney showed that the pre-treatment of miR-138 antagomir decreased the infiltration of inflammatory cells and damaged the tissue structure compared with that in the control group (Fig. 3A). ELISA showed that the levels of the inflammatory factors, IL-1 $\beta$, TNF- $\alpha$ and IL-6, were all decreased by miR-138 antagomir (Fig. 3B). Additionally, the levels of Cr, BUN, AST and ALT in serum from the miR-138 antagomir group were all decreased compared with those in the control group, representing a protective effect of renal and hepatic function by miR-138 antagomir pre-treatment (Fig. 3C, 3D). Thus, miR-138 may participate in the inflammatory process, and the antagomir of miR-138 could alleviate this process in mice models, providing a potential strategy. 
Fig. 3. MiR138 antagomir alleviated tissue damage and organ dysfunction in a sepsis animal model. A-D, Mice were treated with miR-138 antagomir (in-138) or control inhibitor (NC) for 48 hours, followed by the LPS challenge. Representative pathological results of the H\&E stain for heart, liver, lung and kidney are shown, and the tissues damage for the four organs were evaluated by a histopathologic scoring system (A). The serum levels of IL-1 $1 \beta$, IL- 6 and TNF- $\alpha$ were measured by ELISA (B), and the levels of Cr, BUN, ALT and AST (C-D) in the serum were tested. The scale bar in A represents $50 \mu \mathrm{m}$. $\mathrm{N}=6$ for each group.

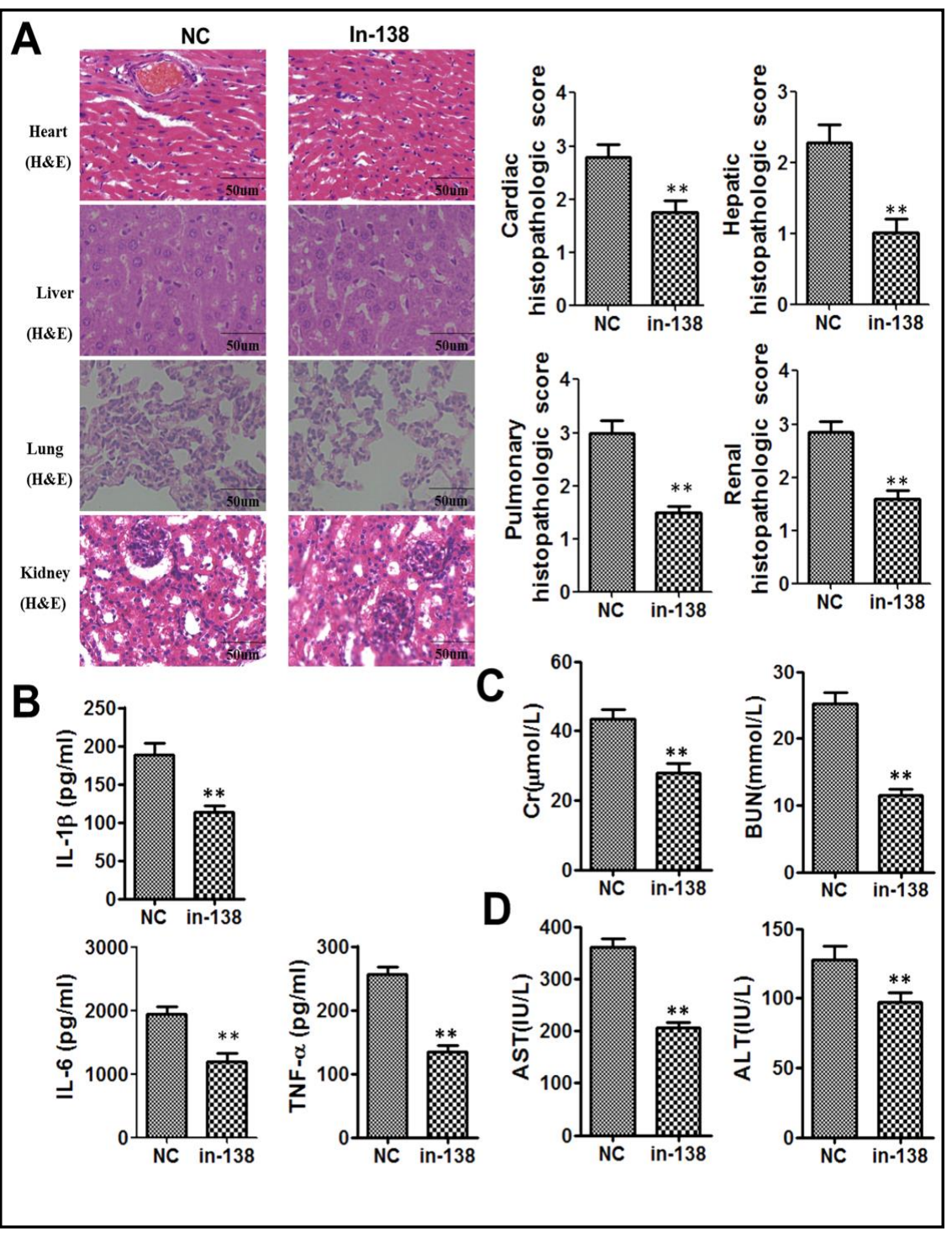
${ }^{*}, \mathrm{p}<0.05 ;{ }^{* *}, \mathrm{p}<0.01$.

\section{SIRT1 is a target of miR-138}

Given the important role of miR-138 in inflammation, we wanted to determine its precise mechanism. Target gene prediction databases were used and SIRT1 was identified as a potential target, showing that the 3'-UTR of SIRT1 mRNA contains a fragment that could be the binding site for miR-138 (Fig. 4A). To further validate the direct interaction of miR138 and SIRT1 3'-UTR, a luciferase reporter system was used. We confirmed the binding of miR-138 to the 3'-UTR of SIRT1 mRNA, showing that the co-transfection of the miR-138 mimic and the SIRT1 WT 3'-UTR significantly decreased the luciferase activity while the plasmid containing a mutation in the SIRT1 3'-UTR or the control plasmid had no effect (Fig. 4B). Then, we transfected mimic or inhibitor of miR-138 into RAW 264.7 macrophages to verify the relationship of miR-138 and SIRT1. The mRNA and protein levels were both downregulated by miR138 mimic and upregulated by the inhibitor (Fig. 4C, 4D). 
Fig. 4. SIRT1 is a target of miR-138 A, The target prediction databases, including TargetScan, PicTar and miRanda, were used, and SIRT1 was identified as a target of miR-138. Sequence alignment of the 3' UTR of SIRT1 show the complementarity at the $5^{\prime}$ end of miR-138. B, luciferase reporter system was used by co-transfecting of miR-138 mimic and SIRT1 3'-UTR (WT) into 293 cells. The

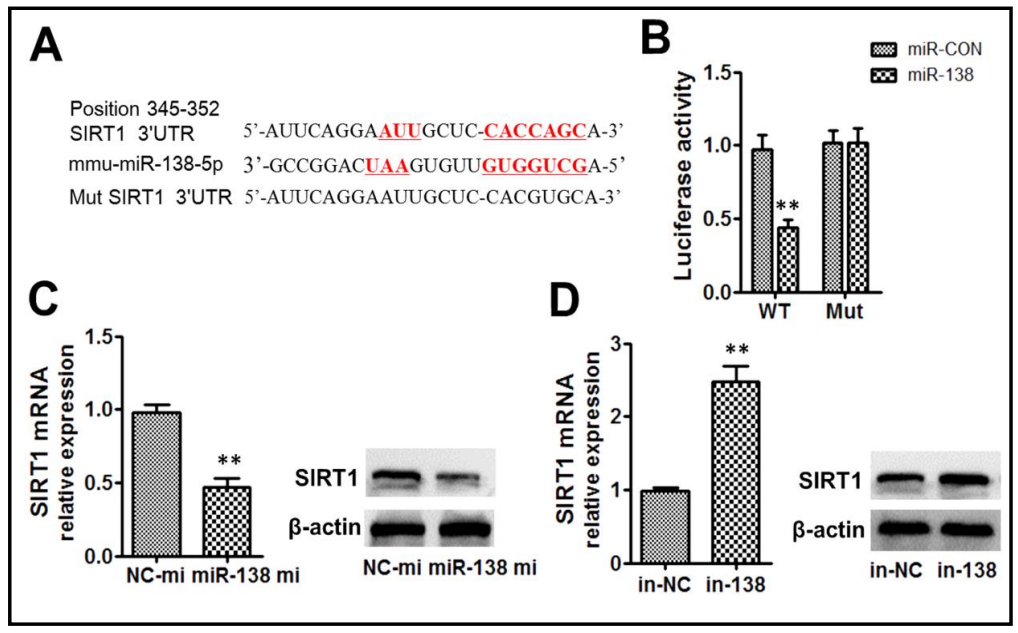
plasmids containing control fragment (miR-CON) or the mutation of SIRT1 3'-UTR (Mut) were used as controls. C-D, The mimic (miR$138 \mathrm{mi}$ ) or inhibitor of miR-138 (in-138) was transfected into RAW 264.7 cells, and the mRNA and protein levels were detected by qRT-PCR and western blotting, respectively. For A-D, The data are the representative results of four independent experiments. NC-mi, normal control mimic. In-NC, inhibitor control. ${ }^{* *}, \mathrm{p}<0.01$.

Fig. 5. MiR-138 participated in macrophage activation by targeting SIRT1 A, the effect of SIRT1 siRNA (siSIRT1) was verified by qRT-PCR. B-D, RAW 264.7 cells were transfected with miR-138 inhibitor (in-138) alone or miR138 inhibitor and si-SIRT1 for 24 hours, and followed by stimulation with LPS. At four hours later, the relative transcript levels for IL-1 $\beta$ (B), IL-6 (C), and TNF- $\alpha$ (D) were detected by qRT-PCR, and the activation of the two pathways, i.e., the AKT and NF- $\kappa$ B pathways, was detected by using western blotting at 30 minutes after LPS stimulation (E). The control inhibitor (in-NC) and control siRNA (si-NC) were used as control. For B-D, the data are the representative results of four independent experiments. ${ }^{* *}, \mathrm{p}<0.01$.

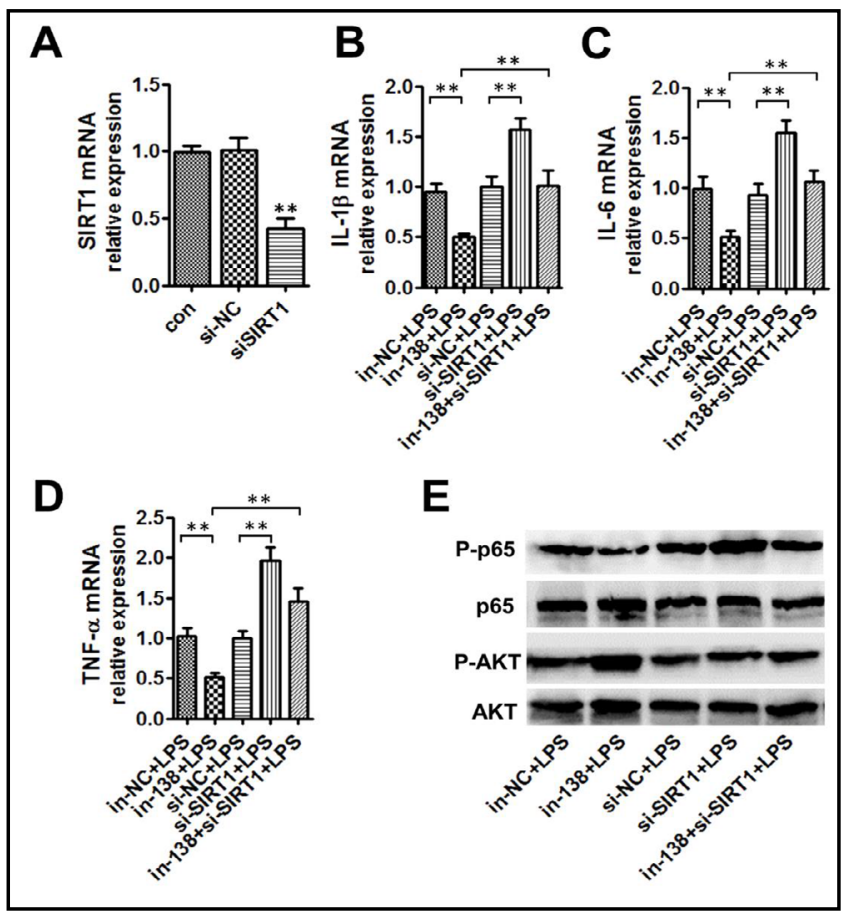

MiR-138 participated in macrophage activation and inflammatory response by targeting SIRT1

To understand whether miR-138 participated in the inflammatory response by targeting SIRT1, we transfected RAW 264.7 macrophages with SIRT1 siRNA (si-SIRT1) to investigate the role of this molecule. The efficacy of siRNA was confirmed by qRT-PCR (Fig. 5A). In macrophages stimulated with LPS, si-SIRT1 significantly elevated the levels of IL-1 $\beta$, IL- 6 and TNF- $\alpha$ compared with those in the control group, confirming its silencing effect (Fig. 5B-D). The silencing of SIRT1 partly reversed the levels of IL-1 $\beta$, IL- 6 and TNF- $\alpha$ that were decreased by the miR-138 inhibitor (Fig. 5B-D). The AKT pathway was alleviated, and NF- $\kappa B$ pathway was re-activated (Fig. 5E). Thus, we demonstrated that miR-138 participated in macrophage 


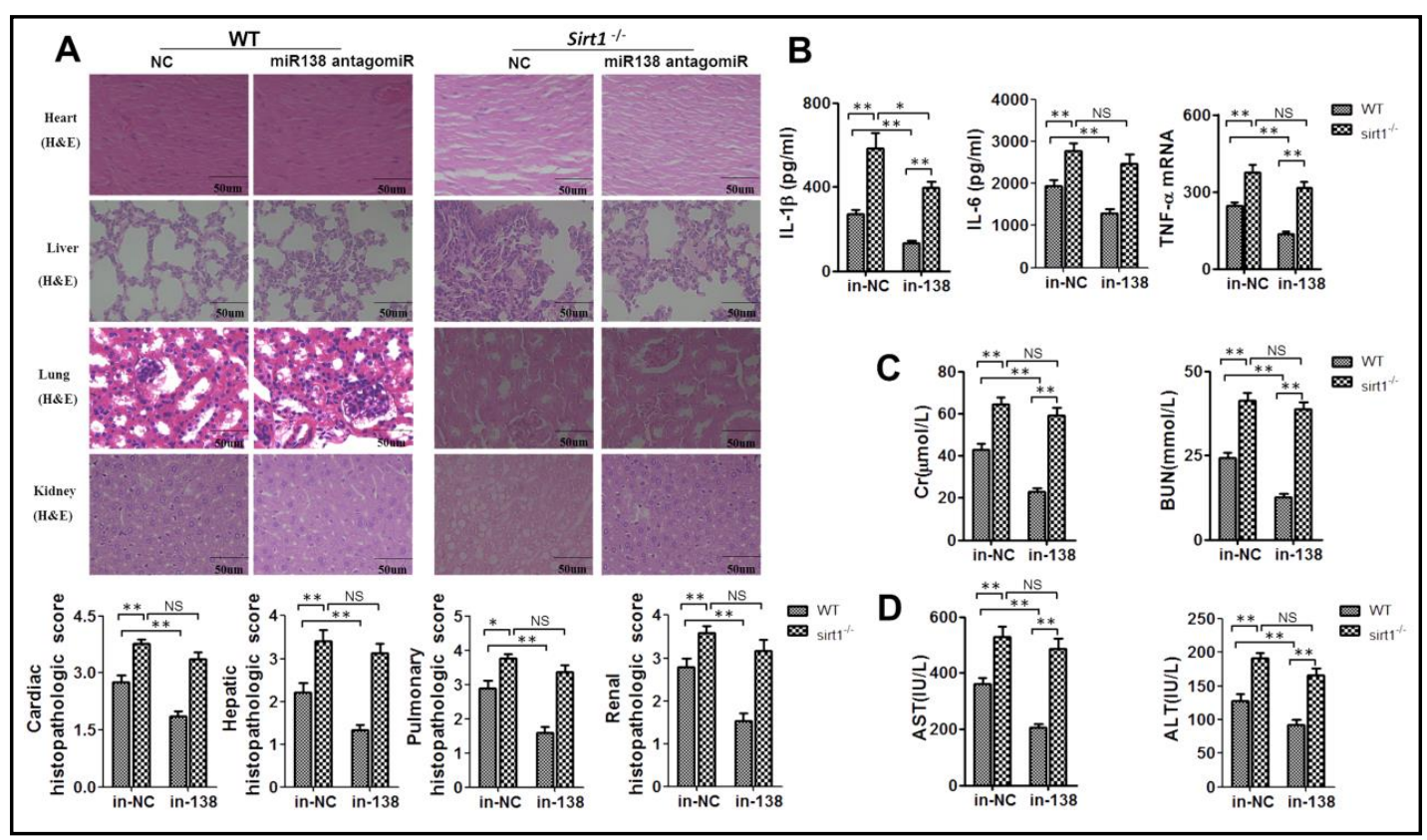

Fig. 6. MiR-138 participated in sepsis process by targeting SIRT1 A-C, C57BL/6 and sirt1-/- mice were treated with miR-138 antagomir (in-138) or control inhibitor (in-NC) for 48 hours, followed by the LPS challenge. Representative pathological results of the H\&E staining of the heart, liver, lungs and kidneys are shown, and the tissues damage for the four organs were evaluated by a histopathologic scoring system (A), the serum level of TNF- $\alpha$, IL- 6 and IL-1 $\beta$ was measured by ELISA (B), and the levels of Cr, BUN, ALT and AST (C-D) in serum were tested. The scale bar in A represents $50 \mu \mathrm{m}$. N=6 for every group. **, $\mathrm{p}<0.01$.

activation by targeting SIRT1, followed by the activation of the NF- $\kappa$ B pathway. To verify this mechanism, we established a model by stimulating sirt $1 \%$ mice with LPS. As shown above, the antagomir of miR-138 significantly alleviated the infiltration of inflammatory cells and structure disorder in heart, liver, lung and kidney tissue. However, these effects disappeared in sirt $1 \%$ mice (Fig. 6A). Additionally, the increased level of inflammatory factors, including IL-1 $\beta$, TNF- $\alpha$ and IL-6 (Fig. 6B), and the biochemical markers, including Cr, BUN, AST and ALT, were all decreased with pre-treatment of miR-138 antagomir and increased in sirt1\% mice (Fig. 6C, 6D). Thus, miR-138 promoted the inflammatory process through its negative control of SIRT1 in macrophages.

\section{Discussion}

In the present study, we identified the role of miR-138 in the inflammatory process. First, we found that miR-138 was increased in LPS-stimulated macrophages. By using the inhibitor of miR-138, we confirmed its effect during macrophage activation induced by LPS. Then, the animal model of inflammation induced by LPS intraperitoneal injection was used and miR-138 was shown to aggravate inflammation, as the pre-treatment of miR-138 antagomir significantly alleviated the tissue damage and organ dysfunction. For a better understanding of the mechanism, we searched the target gene prediction databases and found that SIRT1 might be a potential target of miR-138. After confirmation of the direct binding of miR138 with SIRT1, the SIRT1 inhibitor and sirt1-/- mice were used for further studies. We demonstrated that miR-138 participated in the inflammatory process by targeting SIRT1, and two pathways, including NF- $\kappa \mathrm{B}$ and AKT pathways, were involved (Fig. 7). Thus, the inhibition of miR-138 may be a promising strategy for sepsis therapy. Sepsis often causes severe tissue damage and organ dysfunction owing to the cytokine storm. In the present study, mice were challenged with LPS to induce acute inflammation in vivo. Compared with clinical 
sepsis, this model showed similar inflammation responses, thus we used this model to study the role of miR-138 in inflammation. However, this model doesn't contain immunosuppression followed by inflammation. Therefore, the results still need to be verified before clinical trials. The lungs, kidneys, liver and heart are the most common organs threatened by sepsis $[23,24]$. Owing to the severe inflammation, the pulmonary tissue shows disordered and collapsed alveolar structures and severe infiltration of inflammatory cells [25]. The hepatic tissue shows hepatocytes necrosis and edema in sepsis. Additionally, ALT and AST, which represent the degree of liver injury, are significantly increased $[26,27]$. The kidney is one of the most vulnerable organs in sepsis. The tissue shows glomerulus contraction and dilatation, and edema in renal tubular epithelial and interstitial tissues. The BUN and Cr levels in serum are

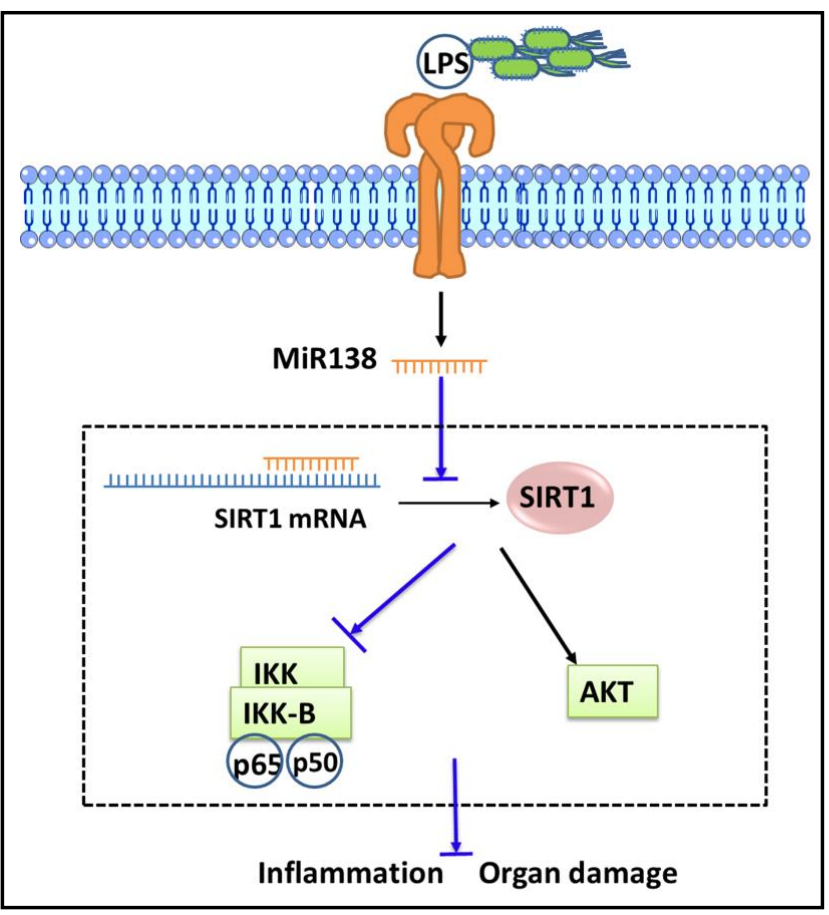

Fig. 7. MiR-138 targets SIRT1 and then activates NF- $\kappa B$ while blocking AKT, leading to the aggravation of organ damage During sepsis, miR-138 was increased in macrophages. By binding to mRNA of SIRT1, miR-138 activates NF- $\kappa B$ and blocks AKT, thus promoting inflammation and organ damage in sepsis. increased, suggesting kidney dysfunction [28]. In the present study, miR-138 was responsible for the tissue damage and organ dysfunction induced by LPS. A previous study showed that miR-138 is increased due to inflammation situation in periodontitis model or in endothelial cells under stimulation with LPS $[29,30]$, but miR-138 is only considered as a consequence of inflammation in these two studies. In the present study, we found that miR-138 not only increased as a result of inflammation but also acted as a regulator by targeting SIRT1, thus providing a potential target for sepsis treatment. However, the antagomir was used before LPS challenge in vivo, which is a pre-treatment model. This model was suitable for studies focusing on the mechanism, but more work is needed to simulate the clinical situation.Macrophage participates in the process of sepsis. M1 macrophages cause a severe cytokine storm, which leads to severe inflammatory reaction and tissue damage, while M2 macrophages are associated with immune suppression [31]. The manifestation of different properties is dependent on the microenvironment. The two phenotypes are extreme statuses representing a series of subtypes, and the inner changes of macrophages rely on several transcription factors and signal pathways, such as STAT1, NF- $\kappa B$, STAT6 or AKT pathways [32, 33]. Recently, miRNAs have been shown to influence macrophage polarization [9]. We found that by targeting SIRT1 in macrophages, miR138 at least partly regulated the activity of the NF-KB pathway, which was significantly decreased after the inhibition of miR-138. This finding was consistent with the decreased mRNA levels of several inflammatory factors. However, the AKT pathway was activated by miR-138 inhibitor, implying the inhibition of miR-138 on the AKT pathway. Previous studies have shown that the AKT pathway also participates in macrophage polarization [10]. In the present study, the increased activation of the AKT pathway was accompanied by decreased inflammatory cytokines, further supporting the idea that AKT participates in the activation of M2 macrophages 34. Additionally, the present study is consistent with recent 


\section{Cellular Physiology Cell Physiol Biochem 2018;49:489-500 \begin{tabular}{l|l|l} 
DOI: 10.1159/000492988 & $\begin{array}{l}\text { O 2018 The Author(s). Published by S. Karger AG, Basel } \\
\text { www.karger.com/cpb }\end{array}$
\end{tabular}}

studies showing that AKT plays a protective role in sepsis [35-37]. SIRT1 shows strong anti-inflammation activity [38]. In sepsis, SIRT1 may promote the termination of the NF$\kappa B$-dependent transcription by its deacetylation effect of $\mathrm{p} 65$ and thus play a role in sepsis protection $[6,39]$. Additionally, SIRT1 directly or indirectly regulates the AKT pathway, [40, 41]. Recent studies showed that SIRT1 is regulated by several mi-RNAs including miR-34a, miR-181, miR-217, etc. [42, 43]. MiR-138 directly targets SIRT1 in osteosarcoma, non-small cell lung cancer and axon regeneration [16, 20,21]. The inhibitor or antagomir of miR-138 protected mice from the inflammatory reaction, tissue damage and organ dysfunction, and the $\mathrm{NF}-\mathrm{\kappa B}$ pathway was inhibited while the AKT pathway was activated. However, after SIRT1 was inhibited or knocked out in macrophages, the protection effect disappeared, and the $\mathrm{NF}-\kappa \mathrm{B}$ pathway was activated and the AKT pathway was inhibited again. Thus, miR-138 promoted inflammation through the miR-138-SIRT1-NF- $\kappa B$ /AKT axis. This study provides the first evidence that miR-138 and miR-138-SIRT1 are involved in sepsis. In conclusion, we demonstrated the important role of miR-138 in the inflammatory responses of macrophages in LPS-challenged mice. We found that miR-138 promoted inflammatory reactions, tissue damage and organ dysfunction, which depends on the direct targeting of SIRT1 by miR-138. Specifically, miR-138 bound to and inhibited the activity of SIRT1 mRNA, followed by the activation of the $N F-\kappa B$ pathway and the inhibition of the AKT pathway in macrophages, thus promoting inflammation. The downregulation of miR-138 by its inhibitor could reverse this process by upregulating SIRT1, terminating NF- $\mathrm{BB}$ and activating the AKT pathway. The present study provides a promising miRNA target for sepsis therapeutic strategies.

\section{Acknowledgements}

This work was financially supported by grants from the Project of National Natural Science Foundation of China [Nos. 81501663, 81501666, and 81530064]. The authors would like to thank the Burns Center of PLA, Department of Burns and Cutaneous Surgery, Xijing Hospital for support of the present study. The authors would also like to thank the Fourth Military Medical University for providing experimental animals. X. Bai, Y. Liu, and D. Hu reviewed articles and designed the research; X. Bai, JL. Zhang, Y. Liu, M. Cao and JN. Zhang performed experiments; X. Bai, JL. Zhang and Y. Liu wrote the manuscript; W. Zhang, X. Li, K. Wang worked on the statistical analysis; W. Zhang, X. Li, K. Wang, M. Cao, JN. Zhang, F. Han and J. Shi contributed reagents, materials and analysis tools; X. Bai, and D. Hu revised and approved the final submission. X. Bai, Y. Liu, and D. Hu provided funding.

\section{Disclosure Statement}

The authors confirm that there are no conflicts of interest.

\section{References}

$>1$ Stevenson EK, Rubenstein AR, Radin GT, Wiener RS, Walkey AJ: Two decades of mortality trends among patients with severe sepsis: A comparative meta-analysis*. Crit Care Med 2014;42:625-631.

-2 Hotchkiss RS, Moldawer LL, Opal SM, Reinhart K, Turnbull IR, Vincent J: Sepsis and septic shock. Nat Rev Dis Primers 2016;2:16045.

3 Martin GS: Sepsis, severe sepsis and septic shock: Changes in incidence, pathogens and outcomes. Expert Rev Anti Infect Ther 2012;10:701-706.

4 Zheng Y, Zhu D: Molecular hydrogen therapy ameliorates organ damage induced by sepsis. Oxid Med Cell Longev 2016;2016:5806057.

5 Wynn TA, Chawla A, Pollard JW: Macrophage biology in development, homeostasis and disease. Nature 2013;496:445-455. 


\section{Cellular Physiology Cell Physiol Biochem 2018;49:489-500 \begin{tabular}{ll|l} 
DOI: 10.1159/000492988 & O 2018 The Author(s). Published by S. Karger AG, Basel \\
wwww.karger.com/cpb
\end{tabular}

6 van der Poll T, van de Veerdonk FL, Scicluna BP, Netea MG: The immunopathology of sepsis and potential therapeutic targets. Nat Rev Immunol 2017;17:407-420.

7 Murray PJ, Wynn TA: Protective and pathogenic functions of macrophage subsets. Nat Rev Immunol 2011;11:723-737.

-8 Wang S, Li Z, Chen Q, Wang L, Zheng J, Lin Z, Li W: NF-kappaB-Induced MicroRNA-211 inhibits interleukin-10 in macrophages of rats with Lipopolysaccharide-Induced acute respiratory distress syndrome. Cell Physiol Biochem 2018;45:332-342.

-9ssandoh K, Li Y, Huo J, Fan G: MiRNA-Mediated macrophage polarization and its potential role in the regulation of inflammatory response. Shock 2016;46:122-131.

-10 Murray PJ, Allen JE, Biswas SK, Fisher EA, Gilroy DW, Goerdt S, Gordon S, Hamilton JA, Ivashkiv LB, Lawrence T, Locati M, Mantovani A, Martinez FO, Mege J, Mosser DM, Natoli G, Saeij JP, Schultze JL, Shirey KA, Sica A, et al.: Macrophage activation and polarization: Nomenclature and experimental guidelines. Immunity 2014;41:14-20.

11 Mohr AM, Mott JL: Overview of microRNA biology. Semin Liver Dis 2015;35:3-11.

12 Giza DE, Fuentes-Mattei E, Bullock MD, Tudor S, Goblirsch MJ, Fabbri M, Lupu F, Yeung SJ, Vasilescu C, Calin GA: Cellular and viral microRNAs in sepsis: Mechanisms of action and clinical applications. Cell Death Differ 2016;23:1906-1918.

13 Yin Y, Li F, Shi J, Li S, Cai J, Jiang Y: MiR-146a regulates inflammatory infiltration by macrophages in Polymyositis/Dermatomyositis by targeting TRAF6 and affecting IL-17/ICAM-1 pathway. Cell Physiol Biochem 2016;40:486-498.

14 Sun XX, Zhang SS, Dai CY, Peng J, Pan Q, Xu LF, Ma XL: LukS-PV-Regulated MicroRNA-125a-3p promotes THP-1 macrophages differentiation and apoptosis by Down-Regulating NF1 and bcl-2. Cell Physiol Biochem 2017;44:1093-1105.

15 Xu C, Fu H, Gao L, Wang L, Wang W, Li J, Li Y, Dou L, Gao X, Luo X, Jing Y, Chim CS, Zheng X, Yu L: BCR-ABL/ GATA1/miR-138 mini circuitry contributes to the leukemogenesis of chronic myeloid leukemia. Oncogene 2014;33:44-54.

16 Liu CM, Wang RY, Saijilafu, Jiao ZX, Zhang BY, Zhou FQ: MicroRNA-138 and SIRT1 form a mutual negative feedback loop to regulate mammalian axon regeneration. Genes Dev 2013;27:1473-1483.

17 Rehan L, Laszki-Szczachor K, Sobieszczanska M, Polak-Jonkisz D: SIRT1 and NAD as regulators of ageing. Life Sci 2014;105:1-6.

18 Martinez-Pastor B, Mostoslavsky R: Sirtuins, metabolism, and cancer. Front Pharmacol 2012;3:22.

19 Bai X, He T, Gao J, Liu Y, Liu J, Han S, Li Y, Shi J, Han J, Tao K, Xie S, Wang H, Hu D: Melatonin prevents acute kidney injury in severely burned rats via the activation of SIRT1. Sci Rep 2016;6:32199.

20 Ye Z, Fang B, Pan J, Zhang N, Huang J, Xie C, Lou T, Cao Z: MiR-138 suppresses the proliferation, metastasis and autophagy of non-small cell lung cancer by targeting Sirt1. Oncol Rep 2017;37:3244-3252.

21 Yuan Z, Mo H, Mo L, He J, Wu Z, Lin X: Suppressive effect of microRNA-138 on the proliferation and invasion of osteosarcoma cells via targeting SIRT1. Exp Ther Med 2017;13:3417-3423.

-22 Hou LC, Qin MZ, Zheng LN, Lu Y, Wang Q Peng DR, Yu XP, Xin YC, Ji GL, Xiong LZ: Severity of sepsis is correlated with the elevation of serum high-mobility group box 1 in rats. Chin Med J (Engl) 2009;122:449454 .

-23 Gotts JE, Matthay MA: Sepsis: Pathophysiology and clinical management. BRIT MED J 2016;353:i1585.

24 Bejan C, Loghin I, Rosu F, Dorobat G, Dorobat CM: Clinical features and evolution of organ dysfunctions in sepsis. Rev Med Chir Soc Med Nat Iasi 2014;118:71-74.

25 Monti G, Landoni G, Taddeo D, Isella F, Zangrillo A: Clinical aspects of sepsis: An overview. Methods Mol Biol 2015;1237:17-33.

26 Yan J, Li S, Li S: The role of the liver in sepsis. Int Rev Immunol 2014;33:498-510.

27 Bauer M, Press AT, Trauner M: The liver in sepsis: Patterns of response and injury. Curr Opin Crit Care 2013;19:123-127.

28 Martensson J, Bellomo R: Sepsis-Induced acute kidney injury. Crit Care Clin 2015;31:649-660.

29 Zhou X, Luan X, Chen Z, Francis M, Gopinathan G, Li W, Lu X, Li S, Wu C, Diekwisch TG: MicroRNA-138 Inhibits Periodontal Progenitor Differentiation under Inflammatory Conditions. J Dent Res 2016;95:230237.

-30 Sen A, Most P, Peppel K: Induction of microRNA-138 by pro-inflammatory cytokines causes endothelial cell dysfunction. Febs Lett 2014;588:906-914. 


\section{Cellular Physiology Cell Physiol Biochem 2018;49:489-500

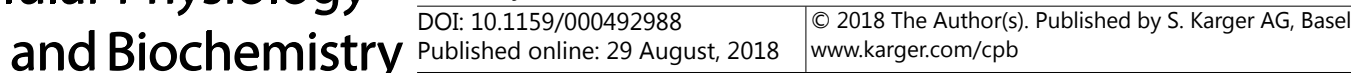

Bai et al.: MicroRNA-138 Promotes Inflammation Through SIRT1

31 Liu YC, Zou XB, Chai YF, Yao YM: Macrophage polarization in inflammatory diseases. Int J Biol Sci 2014;10:520-529.

-32 Glass CK, Natoli G: Molecular control of activation and priming in macrophages. Nat Immunol 2016;17:2633.

-33 Lawrence T, Natoli G: Transcriptional regulation of macrophage polarization: Enabling diversity with identity. Nat Rev Immunol 2011;11:750-761.

34 Rocher C, Singla DK: SMAD-PI3K-Akt-mTOR pathway mediates BMP-7 polarization of monocytes into M2 macrophages. Plos One 2013;8:e84009.

35 Luo K, Long H, Xu B, Luo Y: Metallothionein ameliorates burn sepsis partly via activation of Akt signaling pathway in mice: A randomized animal study. World J Emerg Surg 2015;10:53.

36 Wang GB, Ni YL, Zhou XP, Zhang WF: The AKT/mTOR pathway mediates neuronal protective effects of erythropoietin in sepsis. Mol Cell Biochem 2014;385:125-132.

37 An R, Zhao L, Xi C, Li H, Shen G, Liu H, Zhang S, Sun L: Melatonin attenuates sepsis-induced cardiac dysfunction via a PI3K/Akt-dependent mechanism. Basic Res Cardiol 2016;111:8.

38 Herranz D, Serrano M: SIRT1: Recent lessons from mouse models. Nat Rev Cancer 2010;10:819-823.

-39 Vachharajani VT, Liu T, Wang X, Hoth JJ, Yoza BK, McCall CE: Sirtuins link inflammation and metabolism. J Immunol Res 2016;2016:8167273.

40 Wang RH, Kim HS, Xiao C, Xu X, Gavrilova O, Deng CX: Hepatic Sirt1 deficiency in mice impairs mTorc2/ Akt signaling and results in hyperglycemia, oxidative damage, and insulin resistance. J Clin Invest 2011;121:4477-4490.

41 Li XH, Chen C, Tu Y, Sun HT, Zhao ML, Cheng SX, Qu Y, Zhang S: Sirt1 promotes axonogenesis by deacetylation of Akt and inactivation of GSK3. Mol Neurobiol 2013;48:490-499.

-42 Choi SE, Kemper JK: Regulation of SIRT1 by microRNAs. Mol Cells 2013;36:385-392.

-43 Yamakuchi M: MicroRNA regulation of SIRT1. Front Physiol 2012;3:68. 